\title{
A Legendre Wavelet Spectral Collocation Method for Solving Oscillatory Initial Value Problems
}

\author{
A. Karimi Dizicheh, ${ }^{1}$ F. Ismail, ${ }^{2}$ M. Tavassoli Kajani, ${ }^{3}$ and Mohammad Maleki ${ }^{4}$ \\ ${ }^{1}$ Institute for Mathematical Research, University Putra Malaysia, 43400 Serdang, Selangor, Malaysia \\ ${ }^{2}$ Department of Mathematics, University Putra Malaysia, 43400 Serdang, Selangor, Malaysia \\ ${ }^{3}$ Department of Mathematics, Khorasgan Branch, Islamic Azad University, Khorasgan, Isfahan, Iran \\ ${ }^{4}$ School of Mathematical Sciences, National University of Malaysia (UKM), 43600 Bangi, Selangor, Malaysia
}

Correspondence should be addressed to A. Karimi Dizicheh; ali.karimi@mau.ac.ir

Received 26 January 2013; Revised 15 April 2013; Accepted 15 April 2013

Academic Editor: Srinivasan Natesan

Copyright (C) 2013 A. Karimi Dizicheh et al. This is an open access article distributed under the Creative Commons Attribution License, which permits unrestricted use, distribution, and reproduction in any medium, provided the original work is properly cited.

In this paper, we propose an iterative spectral method for solving differential equations with initial values on large intervals. In the proposed method, we first extend the Legendre wavelet suitable for large intervals, and then the Legendre-Guass collocation points of the Legendre wavelet are derived. Using this strategy, the iterative spectral method converts the differential equation to a set of algebraic equations. Solving these algebraic equations yields an approximate solution for the differential equation. The proposed method is illustrated by some numerical examples, and the result is compared with the exponentially fitted Runge-Kutta method. Our proposed method is simple and highly accurate.

\section{Introduction}

In this paper, we are concerned with the numerical solution of the initial value problem:

$$
F\left(x, y(x), y^{\prime}(x), \ldots, y^{(l-1)}(x), y^{(l)}(x)\right)=0, \quad x \in[0, T),
$$

with the initial conditions

$$
y\left(x_{0}\right)=y_{0}, \ldots, y^{(l-1)}\left(x_{0}\right)=y_{0}^{(l-1)},
$$

on a large domain $[0, T)$. This kind of initial value problems appear in many practical life models and are fundamentally important in both theory and applications. It has applications in many branches of science including celestial mechanics, fluid mechanics, heat wave equations, astrophysics, quantum chemistry, and electronics; for example, see [1-4]. Therefore, it is worthwhile to develop numerical methods to approximate their solutions.

Spectral methods are very powerful tools for solving many kinds of differential equations (and recently integral equations) arising in various fields of science and engineering
$[5,6]$. There are two main advantages for spectral methods. One is associated with high accuracy, that is also called "exponential convergence" meaning that the errors are exponentially small. The second advantage is that they can be easily implemented. These are two effective properties which have encouraged many active researchers to use them for different equations. Among many types of spectral methods, collocation methods are specific approaches that are more applicable and widely used. Spectral collocation methods have been used by many authors; for examples, see [7-12].

In recent years, wavelets have found their way into many different fields of theoretical and practical sciences. Many researchers started using various wavelets [13-15] for analyzing problems of high computational complexity. It is proved that wavelets are powerful tools for exploring new problems and solving differential equations. The Haar wavelets are alternative tools that have been studied for similar purposes; see [16-19].

In this paper, we introduce a reliable algorithm based on the Legendre wavelets spectral method to obtain numerical solutions of nonlinear ordinary differential equations on large intervals. There is a difficulty involved with a direct usage 
of spectral methods and their numerical integration for this type of equations. By introducing a new Legendre wavelet interpolant approximation, these difficulties rest mainly with the improved Legendre waveletspectral method. The method of this paper solves the problem iteratively. The validation of this technique is especially tested for solutions having oscillatory behavior. Numerical examples are included to demonstrate the efficiency and high accuracy of the proposed method.

The remainder of the paper is organized as follows. In Section 2, we explain the basic properties of the Legendre wavelets and Legendre polynomials. The family of the Legendre wavelet is an important example; see Kajani and Vencheh [20]. Then we introduce an interpolation with the Legendre wavelet on shifted Legendre-gauss points and its coefficients. We apply the Legendre wavelets on the spectral method for solving differential equations in large intervals in Section 3. Section 4 is devoted to the numerical results, compared to the exponentially fitted Runge-Kutta method; see Berghe et al. $[21,22]$. Finally, in Section 5, the report ends with a brief conclusion.

\section{The Legendre Wavelets Spectral Method}

In this section, we describe spectral method on the Legendre wavelets. First, the Legendre wavelets on large domain and then spectral method are briefly introduced. Next, we state how the interpolation can be performed using the Legendre wavelets.

2.1. Review of Legendre Wavelets and Legendre Polynomials. Continuous wavelets transform (see [20]) is defined by

$$
\psi_{a, b}(t)=|a|^{-1 / 2} \psi\left(\frac{t-b}{a}\right), \quad a, b \in \mathbb{R}, a \neq 0,
$$

for any continuous function $\psi$, where $a$ is called the scale and $b$ is referred by the shift. A discrete wavelet transform is given by

$$
\psi_{m, n}(t)=a^{-m / 2} \psi\left(a^{-m} t-n b\right),
$$

in which the scale and shift are replaced by $\left(a^{m}, n a^{m} b\right)$ for any $m, n \in \mathbb{Z}$. Here, we intend to consider a family of discrete wavelets $\psi_{m, n}(t)$ when $\psi$ is derived from a Legendre polynomial, and it depends on five arguments, namely, $(k, T, n, m, t) ; T$ is a large integer, $m$ is the order of the Legendre polynomial, $t$ stands for the normalized time,

$$
k=1,2, \ldots, T, \quad n=1,2, \ldots, 2^{k-1} T .
$$

Therefore, we define the Legendre wavelets on the interval $[0, T)$ by

$$
\begin{aligned}
& \psi_{n, m}(t) \\
& = \begin{cases}\left(m+\frac{1}{2}\right)^{1 / 2} 2^{k / 2} L_{m}\left(2^{k} t-2 n+1\right) & \frac{n-1}{2^{k-1}} \leq t<\frac{n}{2^{k-1}}, \\
0 & \text { otherwise, }\end{cases}
\end{aligned}
$$

where $L_{m}(t)$ is the famous $m$ th-order Legendre polynomial with the orthogonal weight function $w(t)=1$. An orthonormal set of the Legendre wavelets is presented in [20]. The Legendre polynomials are obtained by the recursive formulas:

$$
\begin{gathered}
L_{0}(t)=1, \\
L_{1}(t)=t, \\
L_{m+1}(t)=\frac{2 m+1}{m+1} t L_{m}(t)-\frac{m}{m+1} L_{m-1}(t), \quad m=1,2, \ldots
\end{gathered}
$$

Further, the Legendre-Gauss quadrature formula is defined as follows

$$
\int_{-1}^{1} g(x) d x \simeq \sum_{j=0}^{M-1} w_{j} g\left(x_{j}\right)
$$

where the Legendre-Gauss collocation points $x_{0}<x_{1}<$ $\cdots<x_{M-1}$ are the roots of $L_{M}(x)$ in $(-1,1)$ and $\left\{w_{j}\right\}_{j=0}^{M-1}$ are corresponding weights. While explicit formulas for the quadrature nodes are not known, the quadrature weights can be expressed by the following relation:

$$
w_{j}=\frac{2}{\left(1-x_{j}^{2}\right)\left(L_{M}^{\prime}\left(x_{j}\right)\right)^{2}}, \quad j=0,1, \ldots, M-1 .
$$

Thanks to the property of the Legendre-Gauss quadrature formula, it follows that

$$
\begin{aligned}
\int_{a}^{b} f(x) d x & =\frac{b-a}{2} \int_{-1}^{1} f\left(\frac{(b-a) x+a+b}{2}\right) d x \\
& \simeq \frac{b-a}{2} \sum_{j=0}^{M-1} w_{j} f\left(\frac{(b-a) x_{j}+a+b}{2}\right) \\
& =\sum_{j=0}^{M-1} \bar{w}_{j} f\left(\frac{(b-a) x_{j}+a+b}{2}\right),
\end{aligned}
$$

where

$$
\bar{w}_{j}=\frac{(b-a) w_{j}}{2}, \quad j=0, \ldots, M-1 .
$$

For more details about the Legendre polynomials, see [6].

2.2. Interpolation by the Legendre Wavelets. A function $f \in$ $L^{2}[0, T)$ can be expanded in terms of the Legendre wavelets as

$$
f(x)=\sum_{n=1}^{\infty} \sum_{m=0}^{\infty} y_{n, m} \psi_{n, m}(x)
$$

where $\psi_{n, m}(x)$ are the Legendre wavelets defined by (6) and $y_{n, m}$ are given by

$$
y_{n, m}=\int_{(n-1) / 2^{k-1}}^{n / 2^{k-1}} f(t) \psi_{n, m}(t) d t .
$$


Equation (13) can be approximated using the quadrature rule in (8) as follows:

$$
y_{n, m} \simeq \sum_{j=0}^{M-1} \bar{w}_{j} f\left(x_{n j}\right) \psi_{n, m}\left(x_{n j}\right)
$$

where

$$
\begin{gathered}
\bar{w}_{j}=\frac{w_{j}}{2^{k}}, \quad x_{n j}=\frac{x_{j}}{2^{k}}+\frac{2 n-1}{2^{k}}, \\
j=0, \ldots, M-1, \quad n=1, \ldots, 2^{k-1} T .
\end{gathered}
$$

Substituting (13) into (12) gives rise to

$$
f(x)=\int_{(n-1) / 2^{k-1}}^{n / 2^{k-1}} f(t) \sum_{m=0}^{\infty} \sum_{n=0}^{\infty} \psi_{n, m}(x) \psi_{n, m}(t) d t .
$$

Equation (16) gives the "completeness relation" for the basis set, namely,

$$
\sum_{m=0}^{\infty} \sum_{n=0}^{\infty} \psi_{n, m}(x) \psi_{n, m}(t)=\delta(x-t)
$$

and is therefore an identity. If (14) is substituted into (12) (truncated at $M-1$ and $2^{k-1} T$ ) we have

$$
f\left(x_{n i}\right)=\sum_{j=0}^{M-1} f\left(x_{n j}\right) \bar{w}_{j} \sum_{m=0}^{M-1} \sum_{n=0}^{2^{k-1} T} \psi_{n, m}\left(x_{n i}\right) \psi_{n, m}\left(x_{n j}\right) .
$$

The discrete version of (17) is given by

$$
\bar{w}_{j} \sum_{m=0}^{M-1} \sum_{n=0}^{2^{k-1} T} \psi_{n, m}\left(x_{n i}\right) \psi_{n, m}\left(x_{n j}\right)=\delta_{i j} .
$$

Equations (12) (truncated at $M-1$ and $2^{k-1} T$ ), and (14) also supply interpolation functions $I_{n j}(x)$ governed by

$$
\begin{gathered}
I_{n j}(x)=\bar{w}_{j} \sum_{m=0}^{M-1} \psi_{n, m}\left(x_{n j}\right) \psi_{n, m}(x), \\
j=0,1, \ldots, M-1, n=1, \ldots, 2^{k-1} T .
\end{gathered}
$$

In particular, $I_{n j}\left(x_{n i}\right)=\delta_{i j}$. This implies that

$$
f(x) \simeq \sum_{n=1}^{2^{k-1}} \sum_{j=0}^{M-1} I_{n j}(x) f\left(x_{n j}\right),
$$

since the following holds:

$$
\begin{aligned}
& \sum_{n=1}^{2^{k-1} T} \sum_{m=0}^{M-1} \sum_{j=0}^{M-1} \bar{w}_{j} f\left(x_{n j}\right) \psi_{n, m}\left(x_{n j}\right) \psi_{n, m}(x) \\
& =\sum_{n=1}^{2^{k-1} T} \sum_{j=0}^{M-1} \sum_{m=0}^{M-1} \bar{w}_{j} f\left(x_{n j}\right) \psi_{n, m}\left(x_{n j}\right) \psi_{n, m}(x) \\
& =\sum_{n=1}^{2^{k-1} T} \sum_{j=0}^{M-1} \bar{w}_{j} \sum_{m=0}^{M-1} f\left(x_{n j}\right) \psi_{n, m}\left(x_{n j}\right) \psi_{n, m}(x) .
\end{aligned}
$$

\section{Solving IVPs on a Large Domain}

Consider the initial value problem governed by (1) and (2). In order to solve this problem, we divide the interval $[0, T)$ into some subintervals given by $\left[(n-1) / 2^{k-1}, n / 2^{k-1}\right)$ for $n=$ $1, \ldots, 2^{k-1} T$. Consequently, $x \in\left[(n-1) / 2^{k-1}, n / 2^{k-1}\right)$ implies that

$$
\psi_{p j}(x)=0, \quad \text { for any } p \neq n
$$

Thus, $I_{p j}(x)=0$ for all $p \neq n$. As a result, the Legendre wavelet interpolant approximation to the function $y$ on the $n$th subinterval follows (21) and is given by

$$
\begin{aligned}
y(x) & \simeq Y_{n}(x) \\
& =\sum_{p=1}^{2^{k-1}} \sum_{j=0}^{M-1} I_{p j}(x) y_{p j} \\
& =\sum_{j=0}^{M-1} I_{n j}(x) y_{n j}, \quad \text { for } x \in\left[\frac{n-1}{2^{k-1}}, \frac{n}{2^{k-1}}\right) .
\end{aligned}
$$

Inductively, we define that

$$
Y_{n}^{(s)}(x):=\sum_{j=0}^{M-1} I_{n j}^{(s)}(x) y_{n j}, \quad s=1, \ldots, m .
$$

Applying the points $\left\{x_{n j} \mid n=1, \ldots, 2^{k-1} T, j=l, \ldots, M-1\right\}$ into (1) leads to

$$
F\left(x_{n j}, Y_{n}\left(x_{n j}\right), Y_{n}^{\prime}\left(x_{n j}\right), \ldots, Y_{n}^{(l-1)}\left(x_{n j}\right), Y_{n}^{(l)}\left(x_{n j}\right)\right)=0 .
$$

Besides, the initial conditions for the $n$th subinterval can be approximated as follows:

$$
\begin{array}{r}
\lim _{x \rightarrow\left((n-1) / 2^{k-1}\right)^{-}} Y_{n-1}^{(s)}(x)-Y_{n}^{(s)}\left(\frac{n-1}{2^{k-1}}\right)=0, \\
\text { for any } s=0, \ldots, l-1 .
\end{array}
$$

Equations (26) and (27) give a system of $M$ algebraic equations. By solving this algebraic system, we obtain $\left\{y_{n j} \mid\right.$ $\left.n=1, \ldots, 2^{k-1} T, j=0, \ldots, M-1\right\}$. Then, the approximate solution on the $n$th subinterval is obtained by substituting $y_{n j}$ into (24).

Our proposed procedure starts with setting $n=1$. The initial conditions in (2) are used for the approximate solution for the first subinterval $\left[0,1 / 2^{k-1}\right)$. This allows to obtain the initial conditions for the second subinterval from (27). Consequently, the approximate solution of the second subinterval is computed. The procedure is repeated till the last subinterval. Finally, the approximate solution of the IVP in (1) and (2) on the whole domain $[0, T)$ is obtained by

$$
y(x) \simeq \sum_{n=1}^{2^{k-1} T} Y_{n}(x)=\sum_{n=1}^{2^{k-1} T} \sum_{j=0}^{M-1} I_{n j}(x) y_{n j} .
$$


TABLE 1: Numerical comparison for Example 1.

\begin{tabular}{lllc}
\hline Methods & $M$ & $k$ & Errors \\
\hline LWSM & 10 & 4 & $3.30 \times 10^{-15}$ \\
& 12 & 4 & $5.30 \times 10^{-20}$ \\
& 10 & 5 & $7.36 \times 10^{-18}$ \\
EFRKMB & 12 & 5 & $2.85 \times 10^{-23}$ \\
\hline
\end{tabular}

TABLE 2: Numerical comparison for Example 2.

\begin{tabular}{lllc}
\hline Methods & $M$ & $k$ & Errors \\
\hline LWSM & 26 & 3 & $5.69 \times 10^{-14}$ \\
& 27 & 3 & $8.24 \times 10^{-16}$ \\
& 25 & 4 & $2.11 \times 10^{-21}$ \\
& 27 & 4 & $3.48 \times 10^{-24}$ \\
& 20 & 5 & $1.96 \times 10^{-19}$ \\
EFRKMB & 25 & 5 & $8.32 \times 10^{-29}$ \\
\hline
\end{tabular}

\section{Numerical Experiments}

We apply the method presented in the last section and numerically solve five problems to demonstrate the efficiency of the proposed method. We also compare the results with exponentially fitted Runge-Kutta method [21, 22]. Further, the global errors evaluated at the end point of the interval are tabulated. The following notations are used in the tables.

LWSM stands for "the Legendre wavelets on spectral method,"

EFRKMB denotes "the exponentially fitted Runge-Kutta method using Vanden Berghe's technique with $h=0.0625$ "; see $[21,22]$.

Example 1. Consider the initial value problem

$$
\begin{aligned}
& \frac{d^{2} u}{d x^{2}}=-u\left(u^{2}+v^{2}\right)^{-3 / 2}, \\
& \frac{d^{2} v}{d x^{2}}=-v\left(u^{2}+v^{2}\right)^{3 / 2},
\end{aligned}
$$

for $0 \leq x \leq 7$, where $u(0)=1, u^{\prime}(0)=0, v(0)=0, v^{\prime}(0)=1$.

The exact solutions of this problem are $u(x)=$ $\cos (x), v(x)=\sin (x)$. In order to obtain a solid criterion for a global error assessment, we estimate the error at the endpoints. The Euclidean norms of the end-point global errors are provided in Table 1 . Table 1 demonstrates that LWSM gives a better approximation than that of EFRKMB.

Example 2. Let

$$
y^{\prime \prime}=-30 \sin (30 x),
$$

for $0 \leq x \leq 10$, where $y(0)=0, y^{\prime}(0)=1$.

The exact solution of this initial value problem is given by $y(x)=\sin (30 x) / 30$. Table 2 represents the Euclidean norms
TABle 3: Numerical comparison for Example 3.

\begin{tabular}{lllc}
\hline Methods & $M$ & $k$ & Errors \\
\hline LWSM & 12 & 4 & $1.06 \times 10^{-19}$ \\
& 12 & 3 & $1.08 \times 10^{-16}$ \\
& 15 & 3 & $1.95 \times 10^{-24}$ \\
EFRKMB & & & $2.07 \times 10^{-7}$ \\
\hline
\end{tabular}

TABle 4: Numerical comparison for Example 4.

\begin{tabular}{lllc}
\hline Methods & $M$ & $k$ & Errors \\
\hline LWSM & 12 & 4 & $2.06 \times 10^{-18}$ \\
& 12 & 3 & $2.08 \times 10^{-15}$ \\
& 15 & 3 & $2.95 \times 10^{-21}$ \\
EFRKMB & & & $2.40 \times 10^{-9}$ \\
\hline
\end{tabular}

of the numerical errors at the endpoints associated with LWSM and EFRKMB. It indicates that our approach is highly more accurate than EFRKMB in this example. Furthermore, the error decreases for any increase in $M$ and $k$ as it is also the case for Example 1.

Example 3. Let

$$
u^{\prime \prime}=-u+.001 \cos (x),
$$

for $0 \leq x \leq 1000$, where $u(0)=1, u^{\prime}(0)=0$. The exact solution of this initial value problem is $u(x)=\cos (x)+$ $0.0005 x \sin (x)$. The numerical data associated with the endpoint global errors are provided in Table 3 which give us similar conclusions to Examples 1 and 2 as expected.

Example 4. Let

$$
\begin{gathered}
y^{\prime \prime}+y^{3}+y=B \cos (\Omega x), \\
y(0)=0.20042672806900, \\
y^{\prime}(0)=0,
\end{gathered}
$$

where $0 \leq x \leq 300, B=0.002$, and $\Omega=1.01$.

The exact solution is governed by

$$
\begin{aligned}
y(x)= & 0.200179477536 \cos (\Omega x)+0.246946143 \\
& \times 10^{-3} \cos (3 \Omega x)+0.304016 \times 10^{-6} \cos (5 \Omega x) \\
& +0.374 \times 10^{-9} \cos (7 \Omega x) .
\end{aligned}
$$

The numerical comparison of the two methods using the Euclidean norms of the end-point global errors is provided in Table 4. Similar conclusions to those of previous examples are drawn in this table.

\section{Conclusions}

We define an interpolation using the Legendre wavelets for the shifted Legendre-Guass collocation points. Then, this 
gives rise to a set of algebraic equations, and finally its solution constitutes the desired approximate solutions. Our approach is based on a partitioning of the domain interval and solving a given IVP iteratively on each interval. This approach is called an iterative spectral method and is suitable for solving IVP on large intervals.

The main characteristic behind this technique is that it iteratively solves the IVP and the defined interpolation substantially reduces the computational effort. This leads to highly accurate numerical results as depicted by several examples. The proposed method is simple and easy to implement in practical problems. It can be applied for solving other mathematical equations, such as integral equations, integrodifferential equations and partial differential equations.

\section{References}

[1] E. Fehlberg, "Low-order classical Runge-Kutta formulas with stepsize control and their application to some heat transfer problems," NASA Technical Report 315, 1969.

[2] A. D. Raptis and A. C. Allison, "Exponential-fitting methods for the numerical solution of the schrodinger equation," Computer Physics Communications, vol. 14, no. 1-2, pp. 1-5, 1978.

[3] R. B. Berstein, A. Dalgarno, H. Massey, and J. C. Percival, "Thermal scattering of atoms by homonuclear diatomic molecules," Proceedings of the Royal Society of London A, vol. 274, no. 1359, pp. 427-442, 1963.

[4] A. C. Allison, "The numerical solution of coupled differential equations arising from the Schrödinger equation," Journal of Computational Physics, vol. 6, pp. 378-391, 1970.

[5] J. P. Boyd, Chebyshev and Fourier Spectral Methods, Dover, New York, NY, USA, 2nd edition, 2000.

[6] C. Canuto, M. Y. Hussaini, A. Quarteroni, and T. A. Zang, Spectral Methods in Fluid Dynamics, Springer Series in Computational Physics, Springer, New York, NY, USA, 1988.

[7] J. P. Boyd, C. Rangan, and P. H. Bucksbaum, "Pseudospectral methods on a semi-infinite interval with application to the hydrogen atom: a comparison of the mapped Fourier-sine method with Laguerre series and rational Chebyshev expansions," Journal of Computational Physics, vol. 188, no. 1, pp. 5674, 2003.

[8] W. Bao and J. Shen, "A generalized-Laguerre-Hermite pseudospectral method for computing symmetric and central vortex states in Bose-Einstein condensates," Journal of Computational Physics, vol. 227, no. 23, pp. 9778-9793, 2008.

[9] D. Olmos and B. D. Shizgal, "A pseudospectral method of solution of Fisher's equation," Journal of Computational and Applied Mathematics, vol. 193, no. 1, pp. 219-242, 2006.

[10] D. Olmos and B. D. Shizgal, "Pseudospectral method of solution of the Fitzhugh-Nagumo equation," Mathematics and Computers in Simulation, vol. 79, no. 7, pp. 2258-2278, 2009.

[11] C. Canuto, A. Quarteroni, M. Y. Hussaini, and T. A. Zang, Spectral Methods Fundamentals in Single Domains, Scientific Computation, Springer, New York, NY, USA, 2006.

[12] B. D. Shizgal, "Spectral methods based on nonclassical basis functions: the advection-diffusion equation," Computers \& Fluids, vol. 31, no. 4-7, pp. 825-843, 2002.

[13] G. Beylkin, R. Coifman, and V. Rokhlin, "Fast wavelet transforms and numerical algorithms. I," Communications on Pure and Applied Mathematics, vol. 44, no. 2, pp. 141-183, 1991.
[14] C. F. Chen and C. H. Hsiao, "Haar wavelet method for solving lumped and distributedparameter systems," IEE Proceedings Control Theory and Applications, vol. 144, no. 1, pp. 87-94, 1997.

[15] M. Razzaghi and S. Yousefi, "Legendre wavelets direct method for variational problems," Mathematics and Computers in Simulation, vol. 53, no. 3, pp. 185-192, 2000.

[16] H. R. Karimi, "A computational method for optimal control problem of time-varying state-delayed systems by Haar wavelets," International Journal of Computer Mathematics, vol. 83, no. 2, pp. 235-246, 2006.

[17] H. R. Karimi, P. J. Maralani, B. Moshiri, and B. Lohmann, "Haar wavelet-based approach for optimal control of secondorder linear systems in time domain," Journal of Dynamical and Control Systems, vol. 11, no. 2, pp. 237-252, 2005.

[18] H. R. Karimi, B. Moshiri, B. Lohmann, and P. J. Maralani, "Numerically efficient approximations to the optimal control of linear singularly perturbed systems based on Haar wavelets," International Journal of Computer Mathematics, vol. 82, no. 4, pp. 495-507, 2005.

[19] H. R. Karimi, B. Lohmann, P. J. Maralani, and B. Moshiri, "A computational method for solving optimal control and parameter estimation of linear systems using Haar wavelets," International Journal of Computer Mathematics, vol. 81, no. 9, pp. 1121-1132, 2004.

[20] M. T. Kajani and A. H. Vencheh, "Solving linear integro-differential equation with Legendre wavelets," International Journal of Computer Mathematics, vol. 81, no. 6, pp. 719-726, 2004.

[21] G. V. Berghe, H. de Meyer, M. van Daele, and T. van Hecke, "Exponentially-fitted explicit Runge-Kutta methods," Computer Physics Communications, vol. 123, no. 1-3, pp. 7-15, 1999.

[22] G. V. Berghe, H. de Meyer, M. van Daele, and T. van Hecke, "Exponentially fitted Runge-Kutta methods," Journal of Computational and Applied Mathematics, vol. 125, no. 1-2, pp. 107-115, 2000. 


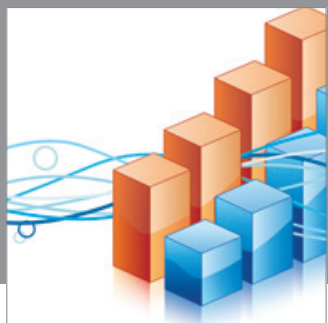

Advances in

Operations Research

mansans

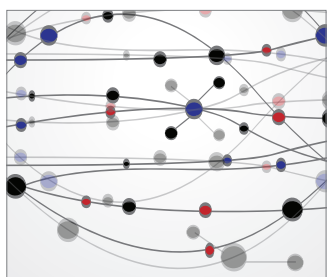

The Scientific World Journal
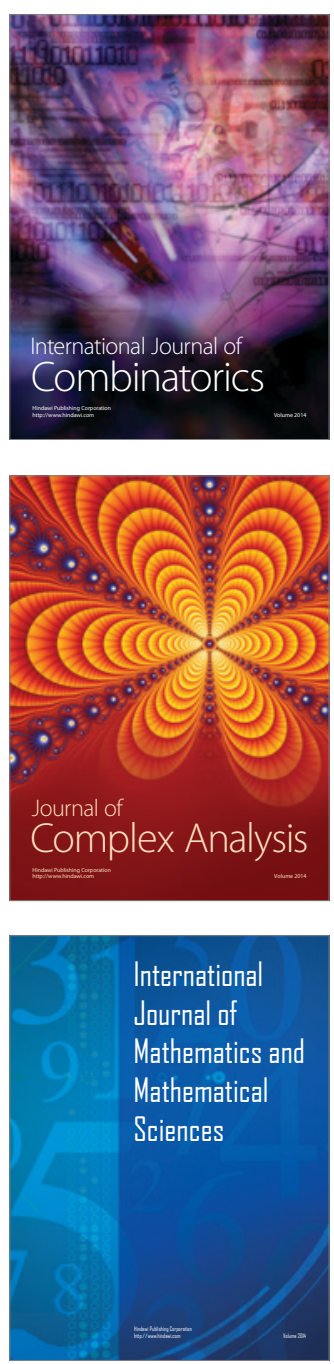
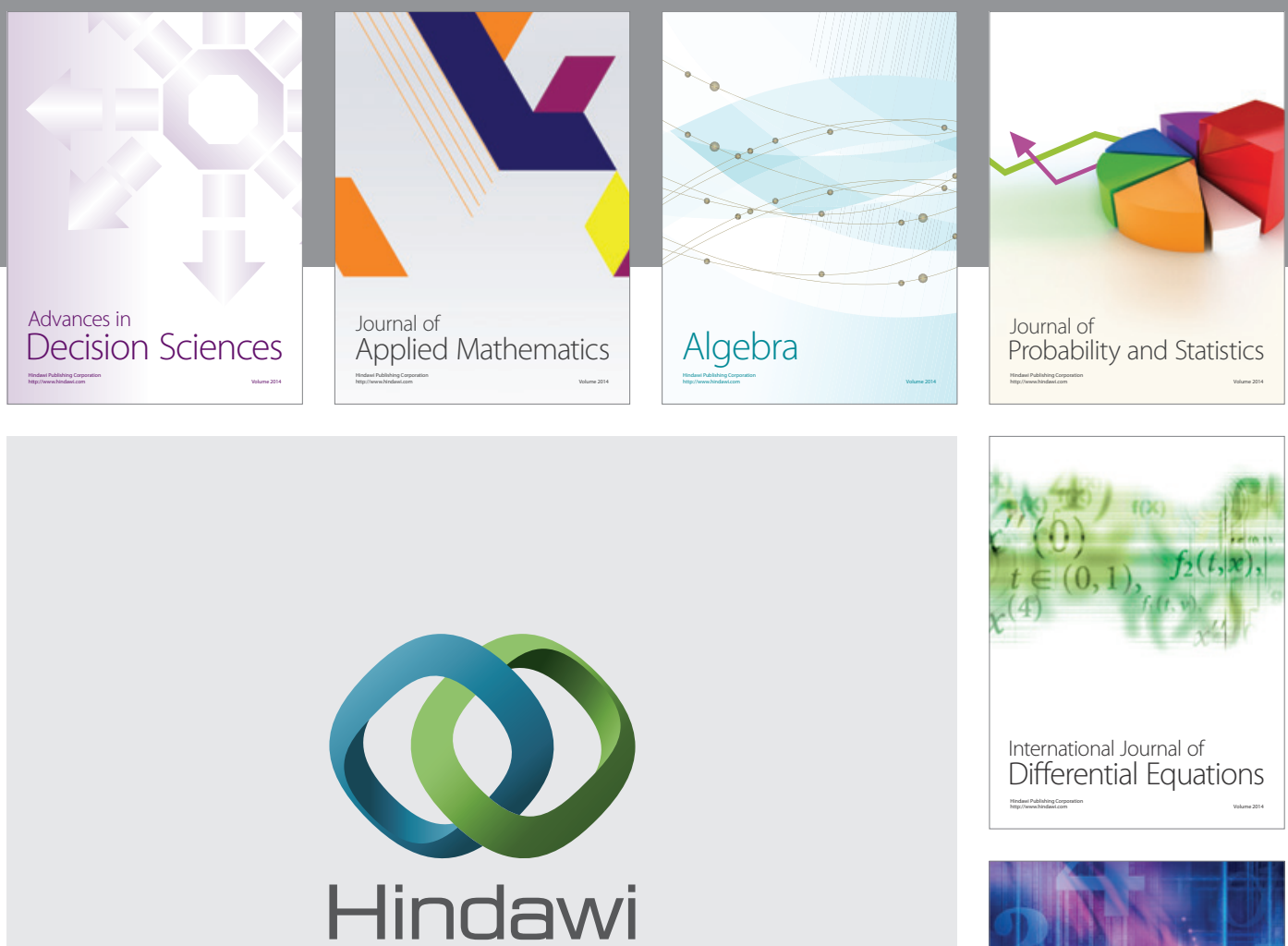

Submit your manuscripts at http://www.hindawi.com
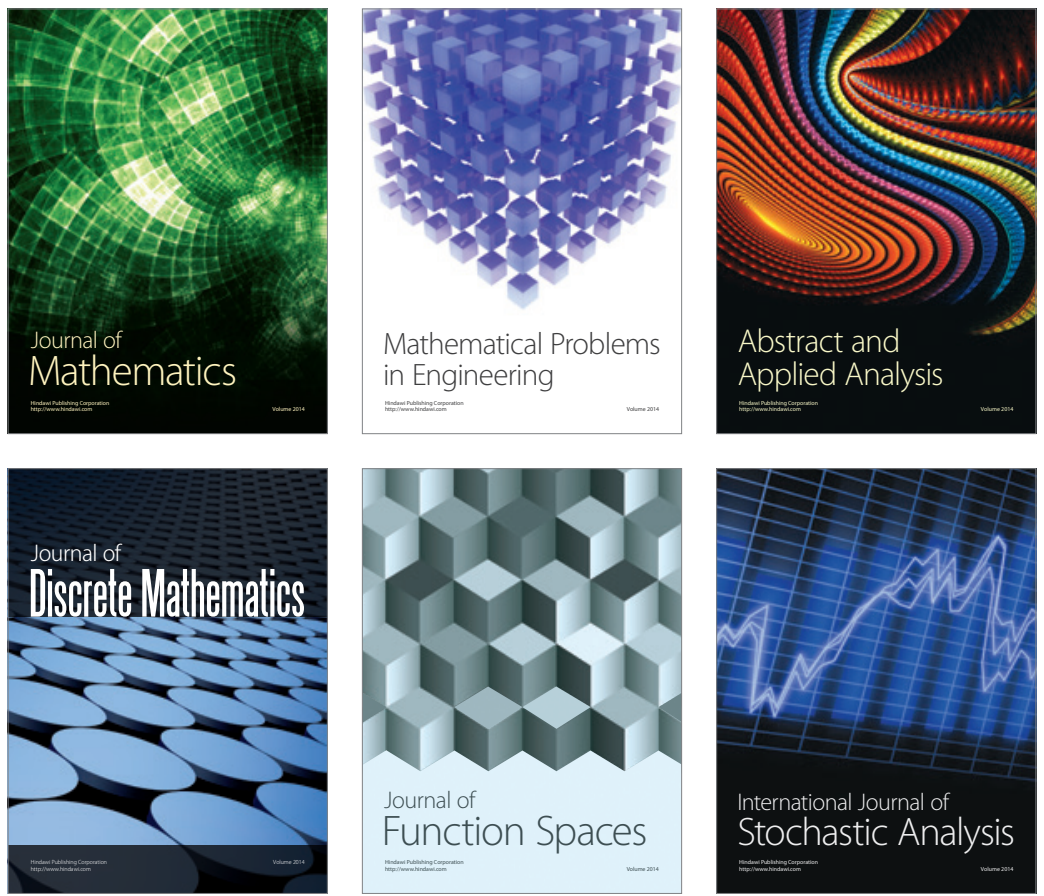

Journal of

Function Spaces

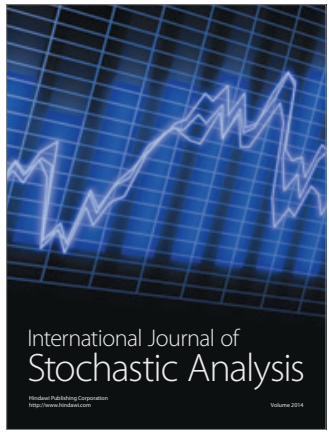

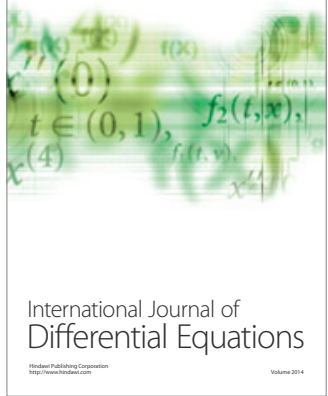
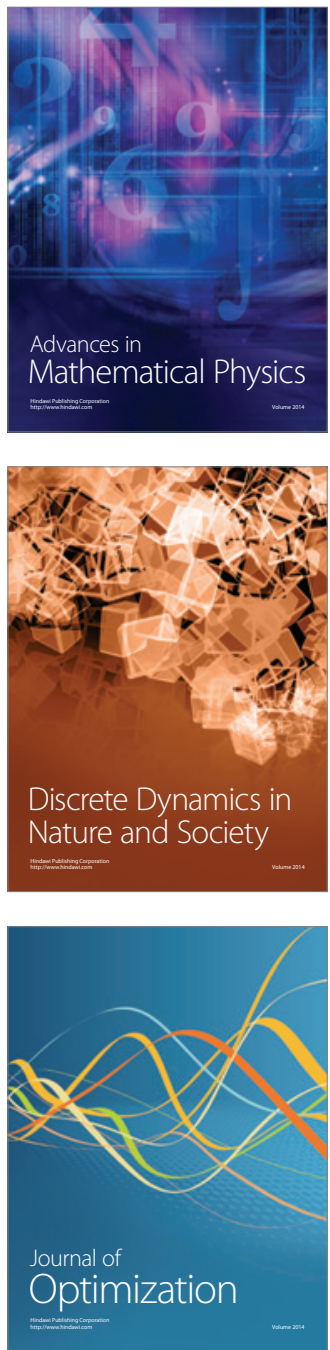